08,09

\title{
Динамика спинорной экситон-поляритонной системы в латерально сжатых GaAs микрорезонаторах при резонансном фотовозбуждении
}

\author{
(C) А.А. Деменев ${ }^{1}$, Н.А. Гиппиус ${ }^{1,2}$, В.Д. Кулаковский ${ }^{1, \uparrow}$ \\ ${ }^{1}$ Институт фризики твердого тела РАН, \\ Черноголовка, Россия \\ ${ }^{2}$ Сколковский институт науки и технологий, ИЦ „Сколково“, \\ Москва, Россия \\ ฯ E-mail: kulakovskulakovs@mail.ru
}

Исследована эволюция пространственной когерентности и поляризации в свободно затухающем поляритонном конденсате, возбуждаемом резонансно на нижнем и верхнем подуровнях нижней поляритонной ветви в микрорезонаторе на основе GaAs с высокой добротностью и пониженной латеральной симметрией линейно поляризованными пикосекундными лазерными импульсами, не приводящими к возбуждению экситонного резервуара. Обнаружено, что конденсат как на нижнем, так и на верхнем подуровне наследует когерентность возбуждающего лазерного импульса в широком диапазоне плотностей возбуждения и сохраняет ее в течение десятков ps. Линейная поляризация фотовозбужденного конденсата сохраняется только в конденсате на нижнем подуровне. Линейно поляризованный конденсат, возбужденный на верхнем подуровне, теряет свою устойчивость при плотностях возбуждения выше некоторого порогового значения: он переходит в режим внутренних джозефсоновских колебаний с сильно осциллирующими циркулярной и диагональной линейной степенями поляризации. При больших плотностях конденсата поляритон-поляритонное взаимодействие приводит к нелинейному эффекту Джозефсона. Все эффекты хорошо описываются в рамках спинорных уравнений Гросса-Питаевского. Показано, что причиной поляризационной неустойчивости конденсата является спиновая анизотропия поляритон-поляритонного взаимодействия.

Работа выполнена при частичной поддержке фонда РФФИ (грант 16-29-0333) и Программы РАН „Наноструктуры: физика, химия, биология, основы технолоийл““.

DOI: $10.21883 /$ FTT.2018.08.46245.06Gr

\section{1. Введение}

Квазидвумерные экситонные поляритоны в полупроводниковых планарных микрорезонаторах (MP) формируются из экситонов и фотонов в условиях их сильного взаимодействия [1]. Как следствие, экситонполяритонные системы демонстрируют коллективные свойства, типичные как для материи, так и для света. Большой интерес к экситон-поляритонным конденсатам в МР в последние годы обусловлен несколькими причинами. Во-первых, поляритонная система является весьма удобной модельной системой для исследования свойств бозе-конденсатов благодаря (i) высокой температуре (десятки и даже сотни К) конденсации вследствие очень малой эффективной массы поляритонов, (ii) возможности контроля величины поляритон-поляритонного взаимодействия путем вариации зазора между экситонным и фотонным резонансом в МР и (iii) возможности прямого определения всех параметров поляритонного конденсата из оптических измерений благодаря наличию фотонной компоненты [2-6]. Кроме того поляритонные системы в отличие от бозе-конденсатных фаз холодных атомов допускают возможность формирования сильно неравновесных макроскопически когерентных состояний, между которыми возможны неравновесные переходы, которыми можно управлять на масштабе микрометров и пико- секунд [7]. Наконец, дополнительная спиновая степень свободы у поляритонов ведет к экстраординарным свойствам как бозе-конденсата, так и его возбуждений, исследование которых представляет интерес не только с академической точки зрения.

Большинство исследований свойств поляритонных конденсатов до настоящего времени проводилось в условиях межзонного возбуждения [8-11]. В этих условиях возбуждаются свободные электроны и дырки в квантовых ямах в активной области МР, которые сначала связываются в долгоживущие экситоны. Конденсированное состояние поляритонов возникает вследствие рассеяния экситонов в состояния с малыми квазиимпульсами $k$ на нижней поляритонной ветке и формированию макрозаполненного состояния на ее дне при достижении критической плотности поляритонов.

В исследованиях макрозаполненных поляритонных состояний в МР был открыт целый ряд эффектов, схожих с явлениями, присущими бозе-эйнштейновским конденсатам, таких как сверхтекучесть [12], подавление зеемановского расщепления в магнитном поле [13], вихри [14], эффект Джозефсона [15], и др. Следует, однако, отметить, что экситон-поляритонная система в МР является сильно термодинамически неравновесной из-за малых времен жизни поляритонов, которые в доступных в настоящее время МР не превышают сотню пикосекунд, что 
приводит к особенностям в свойствах конденсата. Кроме того, взаимодействие экситон-поляритонного конденсата с плотным экситонным резервуаром, формирующемся при межзонном возбуждении, также влияет на его свойства. Так в исследованиях пространственной когерентности в поляритонных конденсатах при нерезонансном возбуждении было найдено, что она расширяется со скоростью $\sim 10^{8} \mathrm{~cm} / \mathrm{s}[16]$, что на много порядков выше, чем в газе ультрахолодных атомов, в то же время длина когерентности не превышает нескольких десятков $\mu \mathrm{m}$. Основными причинами декогеренции конденсата, кроме ограниченного времени жизни поляритонов, являются потенциальный беспорядок и взаимодействие с экситонным резервуаром $[17,18]$.

Избежать возбуждения экситонного резервуара можно при резонансном возбуждении поляритонной системы ниже энергии экситона $E_{X}$. Большинство исследований поляритонных конденсатов с использованием резонансного фотовозбуждения до сих пор проводилось с использованием импульсов с длительностью, значительно превышающей время жизни поляритонов. В этих условиях свойства конденсата контролируются возбуждающим светом, а конденсат демонстрирует мультистабильное поведение [19-22], обусловленное обратной связью в конденсате со спин-анизотропным поляритон-поляритонным взаимодействием между амплитудой и эффективной резонансной частотой поля в МР [23,24]. Переключения между стационарными состояниями вблизи точек бифуркации сопровождаются резкими скачками напряженности поля и поляризации и приводят к таким явлениям как оптические параметрические осцилляции $[25,26]$, поляритонные солитоны [27-29], спиновые кольца [20,30] и т.д.

В настоящей работе рассматриваются когерентные и поляризационные свойства поляритонных конденсатов в высокодобротных МР со временем жизни поляритонов более десятка пикосекунд при резонансном возбуждении пикосекундными лазерными импульсами. При возбуждении по нормали к плоскости МР такие импульсы создают когерентное макрозаполненное чисто поляритонное состояние с $k=0$, которое постепенно затухает со временем жизни поляритонов. Динамика его когерентных и поляризационных свойств исследуется с использованием время-разрешенных оптических методик.

\section{2. Образец и экспериментальная методика}

В работе исследовался полупроводниковый МР на основе GaAlAs/AlAs наноструктуры, выращенной методом молекулярно-лучевой эпитаксии на GaAs подложке. MP содержал четыре GaAs квантовые ямы толщиной $7 \mathrm{~nm}$, разделенных AlAs барьерами толщиной $4 \mathrm{~nm}$ и находящихся в пучностях электрического поля в активном слое толщиной $\lambda / 2$. Нижнее (верхнее) зеркало состояло из 36 (32) $\mathrm{Al}_{0.2} \mathrm{Ga}_{0.8} \mathrm{As} / \mathrm{AlAs}$ брэгговских слоев. Добротность МР $Q \sim 10^{4}$, расщепление Раби $-10.5 \mathrm{meV}$.
Для исследования спинорного конденсата поляритонов была выбрана область на образце с рассогласованием фотонного и экситонного уровней $E_{\text {cav }}(0)-E_{X}(0)=$ $=-6.5 \mathrm{meV}$. Для измерения сигнала в режиме пропускания в $\mathrm{GaAs}$ подложке было вытравлено окно размером $700 \times 300 \mu \mathrm{m}$, что привело к некоторой анизотропии напряжений в образце и расщеплению основного состояния нижней поляритонной ветки на две моды с ортогональными линейными поляризациями $\pi_{x}$ и $\pi_{y}$. Разность их энергий $\delta_{l}=E_{x}(0)-E_{y}(0)$ лежала в области $0.1 \mathrm{meV}$. Моды поляризованы вдоль сторон вытравленного окна, которые, в свою очередь, параллельны горизонтальной $\left(\pi_{x}\right)$ и вертикальной $\left(\pi_{y}\right)$ осям.

Исследуемый образец находился при температуре около $8 \mathrm{~K}$ в оптическом гелиевом криостате, позволяющем исследовать распределение поляритонов с высоким пространственным $(\sim 1.5 \mu \mathrm{m})$ и угловым $\left(\sim 1^{\circ}\right)$ разрешениями. Для фотовозбуждения экситон-поляритонной системы использовался перестраиваемый Ті-сапфировый лазер (Tsunami, Spectra Physics), генерирующий световые импульсы с длительностью $2-3$ ps и частотой повторения $80 \mathrm{MHz}$. Детектирование сигнала осуществлялось с помощью скоростной камеры (стрик-камеры) с временным разрешением 3 ps. Для записи поляризованных компонент излучения использовались пластинки $\lambda / 4$ и $\lambda / 2$ и поляризаторы.

Для нахождения пространственной корреляционной функции первого порядка $g^{(1)}\left(\mathbf{r}_{1}, \mathbf{r}_{2}, t_{1}-t_{2}=0\right)$, анализировалась интерференция света, испускаемого из разных точек на образце, поскольку амплитуда и фаза электрического поля излучения из МР наследует амплитуду и фазу волновой функции конденсата $\psi[6]$. Временная эволюция интерферограмм измерялась с использованием интерферометра Майкельсона в режиме, обеспечивающем наложение прямого и зеркального изображений, с временным разрешением 3 ps. Наложение было получено путем пропускания луча света через интерферометр Майкельсона, одним из плеч которого была отражающая призма, которая обеспечивала наложение исходного изображения на зеркальное по отношению к вертикальной оси. Разность хода между двумя лучами регулировалась линией задержки. Два световых пучка, проходящих через интерферометр, собирались на детекторе стрик-камеры, расположенной в фокусе объектива. Процедура извлечения $g^{(1)}$ из интерферограм описана в работе [31].

\section{3. Динамика поляризации и пространственной когерентности поляритоннго конденсата, возбужденного на нижнем подуровне}

Большое время жизни экситонных поляритонов в исследованном MP $\left(\tau_{\mathrm{LP}} \sim 20 \mathrm{ps}\right)$ позволяет исследовать когерентные свойства чисто поляритонной системы, 
поскольку резонансное возбуждение поляритонов импульсами длительностью 2 ps (спектральная ширина $\sim 1.2 \mathrm{meV}$ ) ниже энергии экситона на $8 \mathrm{meV}$ не возбуждает экситонный резервуар.

Для возбуждения поляритонов на нижнем подуровне с линейной $\pi_{y}$-поляризацией состояний использовались $\pi_{y}$-поляризованные импульсы. Это состояние остается нижайшим и при его заселении поляритонами, поскольку невозбужденный отщепленный $\pi_{x}$-поляризованный поляритонный подуровень сдвигается вверх по энергии быстрее, чем заполненный нижний, из-за того, что отталкивающее взаимодействие между кросс-поляризованными поляритонами больше, чем между поляритонами с одинаковой линейной поляризацией. Измерения поляризованных спектров излучения поляритонной системы показали, что $\pi_{y}$ поляризация излучения сохраняется в течение длительного времени: степень линейной поляризации уменьшается не более чем на $10 \%$ за 100 ps. Поэтому такую систему можно рассматривать как однокомпонентную.

На рис. 1 показана динамика интенсивности излучения поляритонов $I(t)$, измеренная в MP с отрицательной расстройкой $E_{\mathrm{cav}}-E_{X}=-6.5 \mathrm{meV}$ при возбуждении $\hbar \omega_{p}=E_{\mathrm{LP}}(k=0)+0.3 \mathrm{meV}$ импульсами с энергией $P \sim 0.12 \mathrm{~nJ} /$ pulse в пятне диаметром $d=40 \mu \mathrm{m}$. Как и ожидалось в отсутствие экситонов в экситонном резервуаре, I монотонно уменьшается после окончании импульса накачки. Время жизни поляритонов $\tau_{\mathrm{LP}} \approx 19 \mathrm{ps}$.

Количественная информация о величине корреляционной функции первого порядка $g^{(1)}(x,-x)$ была получена из исследований временных зависимостей интерферограмм излучения конденсата, зарегистрированных с использованием интерферометра Майкельсона.

Зависимости $g^{(1)}(x,-x)$, найденные из набора интерферограмм, измеренных с различными задержками между прямым и зеркальным изображениями, показаны на рис. 1 для нескольких значений $\Delta x=2|x|$ меньше и больше диаметра возбуждающего пятна $d=40 \mu \mathrm{m}$. Видно, что $g^{(1)}$ при $\Delta x<d$ достигает $\sim 0.7$, что близко к предельному значению величины $g^{(1)}=0.87$ при измерении интерферограммы с периодом осцилляций $T_{\mathrm{fr}}=7.5 \mu \mathrm{m}$ при используемом пространственном разрешении $2 \mu \mathrm{m}$. Далее на рис. 1 видно, что заметное уменьшение $g^{(1)}$ становится выраженным только при $t>60 \mathrm{ps}$, когда плотность конденсата $n_{c}$ уменьшается более чем в 100 раз. Такое поведение $g^{(1)}$ в резонансно возбужденном конденсате поляритонов сильно отличается от наблюдаемого ранее в нерезонансно возбужденных MP [16-18] и свидетельствует в пользу высказанных в этих работах предположениях о том, что основной причиной нарушения пространственной когерентности в конденсате является его взаимодействие с экситонным резервуаром.

Для более корректного подтверждения этого вывода мы провели сравнение поведения $g^{(1)}$ при резонансном и нерезонансном возбуждении в одной и той же области MP. Результаты сравнения показали, что в то время

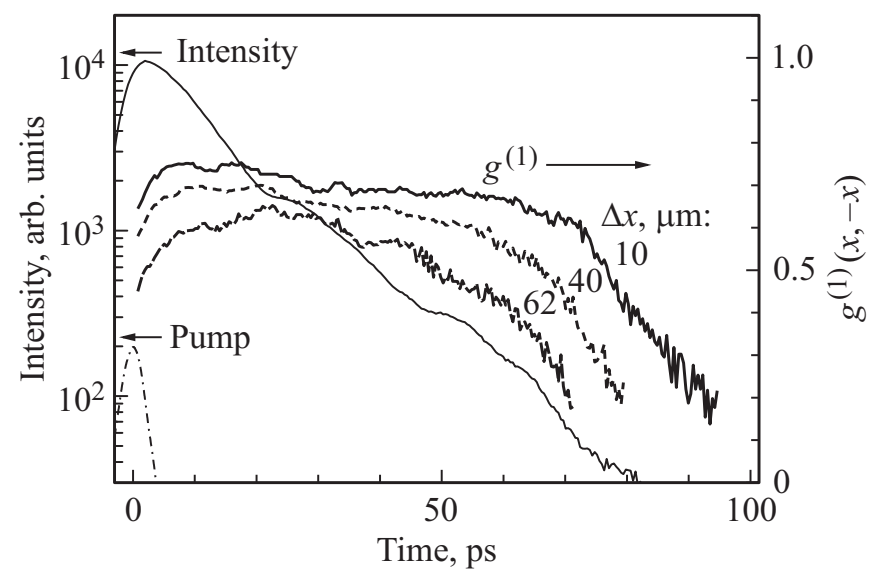

Рис. 1. Временные зависимости интенсивности излучения конденсата (тонкая сплошная линия) и корреляционные функции первого порядка (толстые линии), измеренные в МР при отрицательной расстройке $E_{c}-E_{X}=-6.5 \mathrm{meV}$ и возбуждении $\hbar \omega_{p}=E_{\mathrm{LP}}(k=0)+0.3 \mathrm{meV}$ импульсами с энергией $P \sim 0.12 \mathrm{~nJ} /$ pulse в пятне диаметром $d=40 \mu \mathrm{m}$. Профиль импульса возбуждения показан тонким пунктиром.

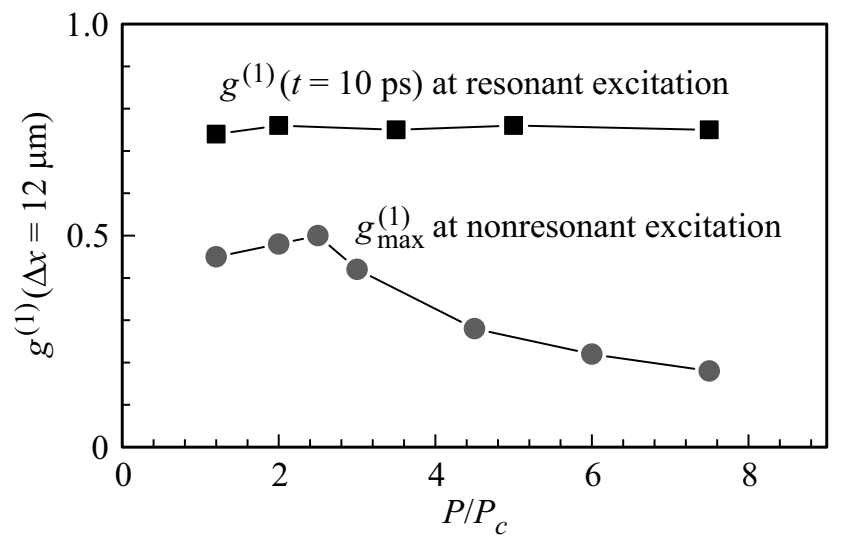

Рис. 2. Зависимости $g^{(1)}(\Delta x=12 \mu \mathrm{m})$ от накачки в резонансно и нерезонансно возбужденных конденсатах. При резонансном фотовозбуждении $g^{(1)}$ измерена при $t=10 \mathrm{ps}$, величина $P_{c}=0.03 \mathrm{~nJ} / \mathrm{pulse}$. Для нерезонансной накачки показаны максимальные значения $g^{(1)}(\Delta x=12 \mu \mathrm{m})$ для приведенных накачек, $P_{c}=P_{\text {thr }}$.

как при резонансном фотовозбуждении $g^{(1)}$ почти не изменяется при вариации плотности возбуждения в 7 раз, при нерезонансном возбуждении $g^{(1)}$ немного растет при увеличении плотности возбуждения выше порога конденсации до $P \sim 2.4 P_{\text {thr }}$ и затем монотонно уменьшается. Результаты сравнения показаны на рис. 2 и 3. В частности, на рис. 2 видно, что при резонансном возбуждении $g^{(1)}(\Delta x=12 \mu \mathrm{m})$ во всей области плотностей возбуждения превышает 0.7, а при нерезонансном возбуждении даже при $P \sim 2.4 P_{\mathrm{thr}}$ величина $g^{(1)}$ не превышает 0.5 и уменышается в 3 раза при $P=7 P_{\mathrm{thr}}$. Из зависимостей же $g^{(1)}$ от $\Delta x$, приведенных на рис. 3, видно, что длина когерентности в резонансно возбужденном конденсате при $t=15$ ps превышает $80 \mu \mathrm{m}$, в то 


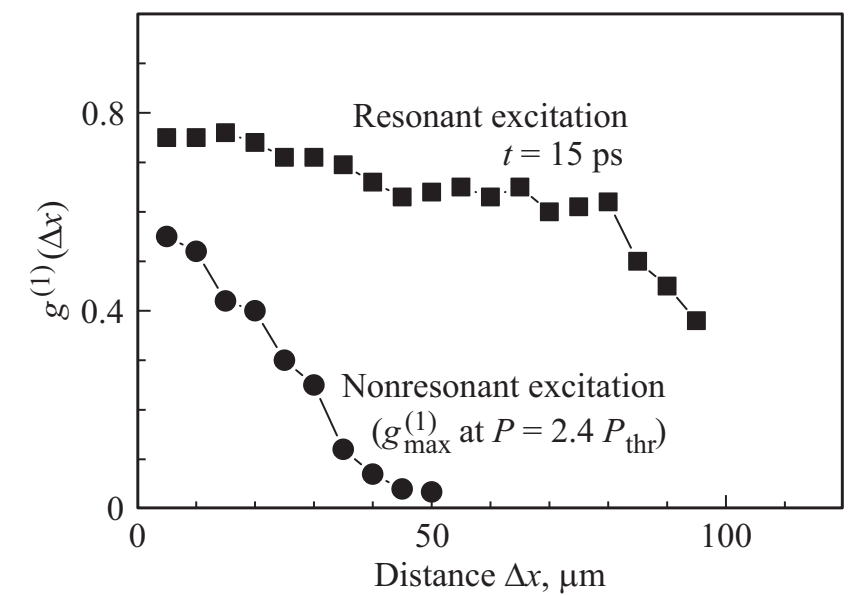

Рис. 3. Зависимости $g^{(1)}(\Delta x)$ при резонансном (квадраты) и нерезонансном (кружки) возбуждениях. Зависимости при резонансном возбуждении измерены при $t=15 \mathrm{ps}$, а при нерезонансном для случая, когда реализуется максимальная длина когерентности.

время как при нерезонансном возбуждении она почти

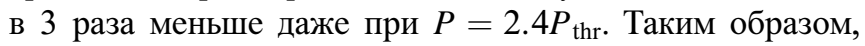
можно сделать однозначный вывод о том, что основной причиной нарушения когерентности поляритонного конденсата при нерезонансном возбуждении является его взаимодействие с плотным экситонным резервуаром.

\section{4. Динамика поляризации и пространственной когерентности поляритонного конденсата, возбужденного на верхнем подуровне}

Для возбуждения поляритонов на верхнем подуровне с линейной $\pi_{x}$-поляризацией состояний использовались $\pi_{x}$-поляризованные импульсы. На рис. 4 приведены временные зависимости суммарной интенсивности излучения поляритонов и ее компонент с разной поляризацией, записанных с разрешением 3 ps при энергии накачки $\sim 2 \mathrm{~nJ} /$ pulse, возбуждении импульсами длительностью 3 ps при нормальном падении луча с малой апертурой $\left(<2^{\circ}\right)$ на MP и $\hbar \omega_{p}=E_{\mathrm{LP}}(k=0)+0.7 \mathrm{meV}$.

На рис. 4, $a$ видно, что $\pi_{x}$-поляризованные импульсы генерируют $\pi_{x}$-поляризованный конденсат. Однако уже при $t \sim 11 \mathrm{ps}$ интенсивность излучения в поляризации $\pi_{y}$ сравнивается с интенсивностью в $\pi_{x}$-поляризации. При дальнейшем увеличении $t$ наблюдаются осцилляции интенсивностей в поляризациях $\pi_{x}$ и $\pi_{y}$, при этом полная интенсивность излучения уменьшается монотонно. Кроме того, на рис. 4, $a$ видно, что при больших $t$ интенсивность излучения в поляризации $\pi_{y}$ становится доминирующей, что свидетельствует о перераспределении поляритонов в спинорной системе в пользу нижнего подуровня. При этом конденсат не переходит в какое-либо определенное стационарное спинорное состояние: на рис. 4, $b$ и 4,c видно, что в диагональных линейных $\left(\pi_{x \pm y}\right)$ и круговых $\left(\sigma^{ \pm}\right)$поляризациях наблюдаются ярко выраженные осцилляции во всем интервале времен.

На рис. 4, $d$ показаны временные зависимости степеней циркулярной $\left(\rho_{c}=\left(I^{+}-I^{-}\right) /\left(I^{+}+I^{-}\right)\right)$, линейных $\left(\rho_{x, y}=\left(I_{x}-I_{y}\right) /\left(I_{x}+I_{y}\right)\right.$ и $\rho_{x \pm y}=\left(I_{x+y}-I_{x-y}\right) /$ $\left.\left(I_{x+y}+I_{x-y}\right)\right)$ поляризаций, а также полной поляризации излучения $\rho_{\Sigma}=\left(\rho_{c}^{2}+\rho_{x, y}^{2}+\rho_{x \mp y}^{2}\right)^{1 / 2}$. На рисунке видно, что в области $t>60 \mathrm{ps}$, в которой плотность конденсата уменьшилась на порядок и межчастичное взаимодействие сильно ослабло, периоды осцилляций $\rho_{c}$ и $\rho_{x \pm y}$ совпадают и остаются неизменными, равными $T=47 \pm 1$ ps. Этот период, как и следовало ожидать, соответствуют величине расщепления уровней $\pi_{x}$ и $\pi_{y}$ в MP: $T=h / \delta_{l}$. Таким образом, мы находим, что после развития поляризационной неустойчивости поляритонный конденсат переходит в режим поляризационных биений, иногда называемых в литературе „внутренними джозефсоновскими осцилляциями“ поляритонов.

При $t<50 \mathrm{ps,} \mathrm{когда} \mathrm{плотность} \mathrm{конденсата} \mathrm{велика,}$ период осцилляций меньше: период первой осцилляции

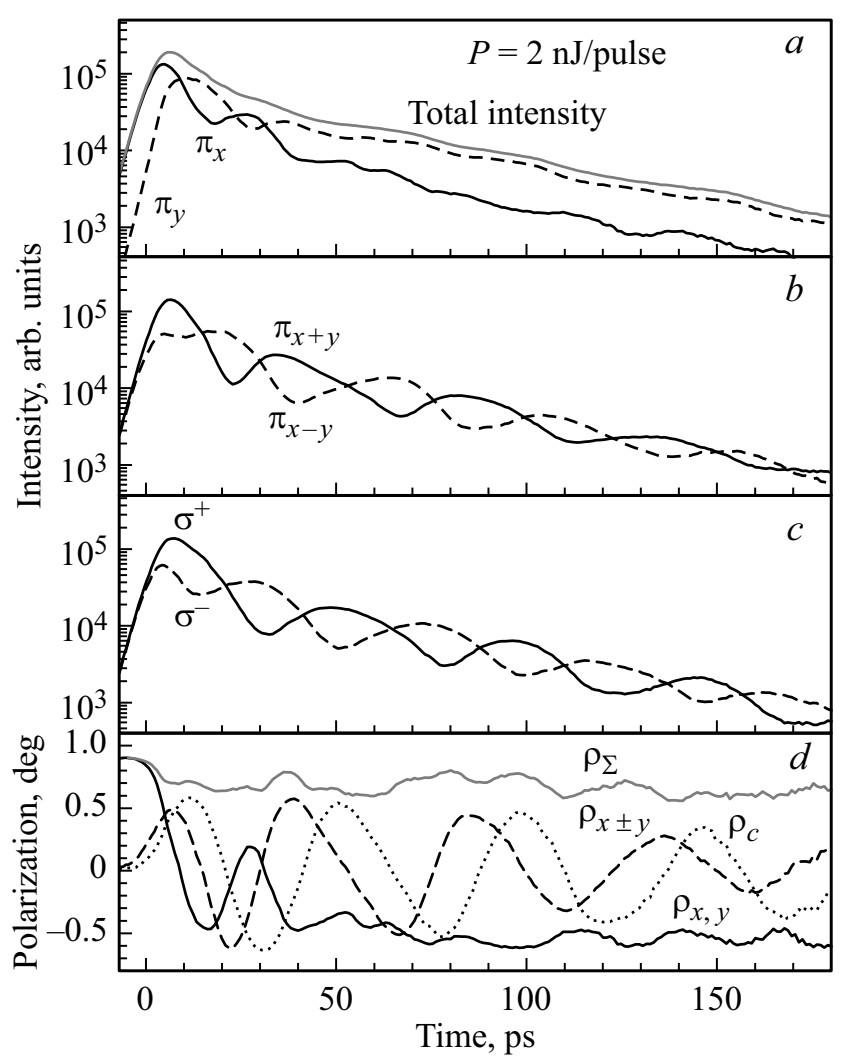

Рис. 4. Временные зависимости суммарной интенсивности излучения поляритонов - $(a)$ и ее компонент с разной поляризацией $(a-c)$, записанных с разрешением 3 ps при энергии накачки $\sim 2 \mathrm{~nJ} /$ pulse, возбуждении $\pi_{x}$-поляризованными импульсами при нормальном падении луча с малой апертурой $\left(<2^{\circ}\right)$ на МР и $\hbar \omega_{p}=E_{\mathrm{LP}}(k=0)+0.7 \mathrm{meV}$. Рис. $4, d$ : зависимости от времени циркулярной $\left(\rho_{c}\right)$, линейных $\left(\rho_{x, y}\right.$ и $\left.\rho_{x \pm y}\right)$ поляризаций, а также полной поляризации излучения $\left(\rho_{\Sigma}\right)$. 


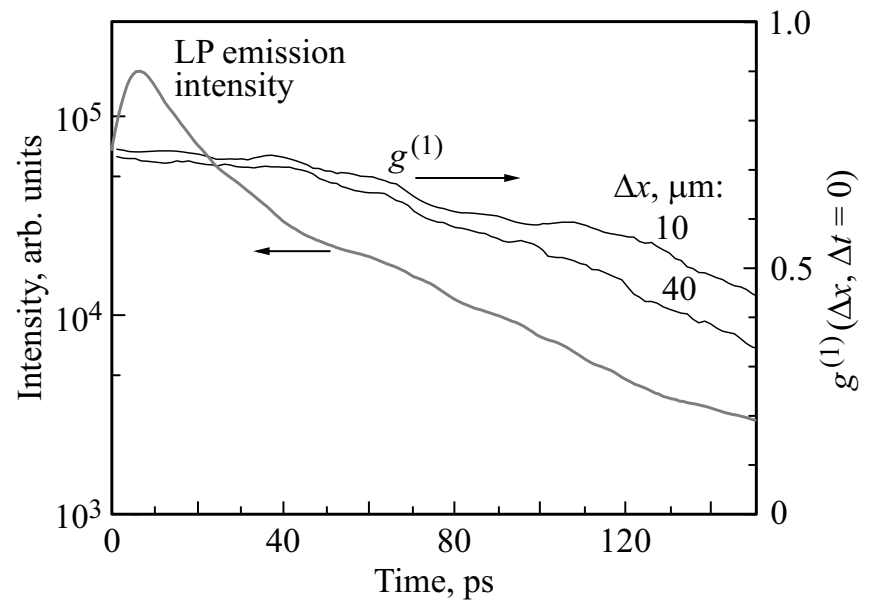

Рис. 5. Зависимости интенсивности излучения поляритонов и корреляционной функции $g^{(1)}$ при $\Delta x=10$ и $40 \mu$ m от времени при возбуждении $\pi_{x}$-поляризованными импульсами длительностью 3 ps с $P \sim 2 \mathrm{~nJ} /$ pulse и $\hbar \omega_{p}=E_{\mathrm{LP}}(k=0)+0.7 \mathrm{meV}$.

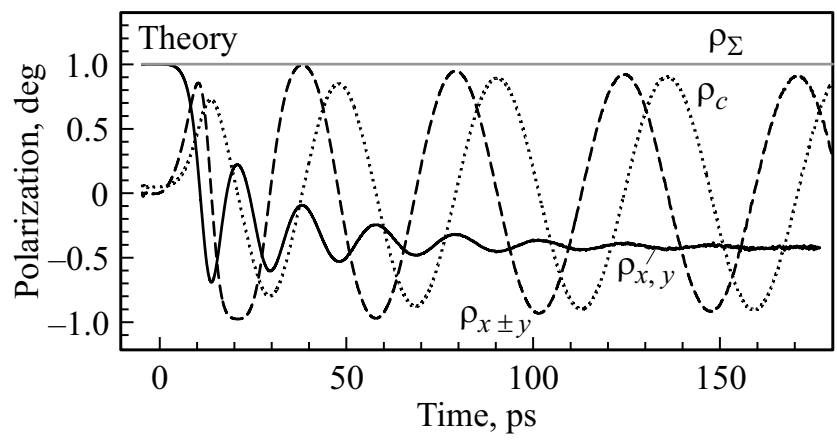

Рис. 6. Рассчитанные зависимости от времени и циркулярной $\left(\rho_{c}\right)$, линейных $\left(\rho_{x, y}\right.$ и $\left.\rho_{x \pm y}\right)$ поляризаций, а также полной поляризации излучения $\left(\rho_{\Sigma}\right)$ с использованием уравнений Гросса-Питаевского и параметров, соответствующих исследуемой системе поляритонов.

в $\rho_{x \pm y}$ равен $32 \pm 2 \mathrm{ps,} \mathrm{что} \mathrm{свидетельствует} \mathrm{о} \mathrm{переходе} \mathrm{в}$ нелинейный режим джозефсоновских осцилляций из-за увеличения расщепления резонансных частот поляритонных состояний, вызванного сильным поляритон-поляритонным взаимодействием. На рис. $4, d$ видно также, что на больших временах $\rho_{x, y}$ меняет знак. Смена знака $\rho_{x, y}$ свидетельствует о перераспределении поляритонов на нижний подуровень. Поскольку все изменения степеней циркулярной и линейных поляризаций происходят при относительно небольшой потере полной поляризации конденсата $\rho_{\Sigma}$, их причиной являются когерентные процессы в конденсате, а не некогерентное рассеяние поляритонов.

Сохранение пространственной когерентности в затухающей спинорной поляритонной системе подтверждается и измерениями корреляционной функции первого порядка. Так, на рис. 5 показаны зависимости $g^{(1)}$ от $t$ при $\Delta x=10$ и $40 \mu \mathrm{m}$ наряду с $I(t)$. Видно, что $g^{(1)}$ в возбужденном пятне уменьшается до 0.5 только при $t>110 \mathrm{ps,}$ когда плотность поляритонов уменьшается почти в 30 раз. На рис. 4 видно, что степень линейной поляризации $\rho_{x, y}$ при $t<110$ ps изменяется от +0.9 до -0.6 , а $\rho_{c}$ и $\rho_{x \pm y}$ демонстрируют 2 периода осцилляций.

Наблюдаемое медленное уменьшение пространственной когерентности подтверждает, что некогерентное рассеяние играет незначительную роль во временной эволюции поляризации конденсата. Это позволяет использовать в качестве первого приближения для описания динамики поляризации резонансно возбужденного поляритонного конденсата модель, основанную на уравнениях Гросса-Питаевского [19,32].

Результаты моделирования эволюции поляризации в свободно затухающем поляритонном конденсате с использованием спинорных уравнений Гросса-Питаевского показаны на рис. 6. Зависимости были рассчитаны с параметрами, соответствующими исследуемой системе поляритонов: $\hbar \omega_{p}=E_{\mathrm{LP}, x}(k=0)+0.7 \mathrm{meV}$, возбуждение по нормали к плоскости MP с почти линейной поляризацией импульса $\left(\rho_{x, y}=0.997, \rho_{c}=0.05\right)$, расщепление $\delta_{l}=80 \mu \mathrm{eV}$ и $\tau_{\mathrm{LP}}=20 \mathrm{ps}$. Поскольку в GaAs MP константа поляритон-поляритонного взаимодейставия для поляритонов с параллельными спинами много больше, чем с антипараллельными, в расчетах предполагалось, что $\alpha_{2}=0$.

Сравнение экспериментальных зависимостей степеней циркулярной и линейных поляризаций, приведенных на рис. 4,c с рассчитанными на рис. 6 показывает, что спинорные уравнения Гросса-Питаевского хорошо описывают все наблюдаемые особенности динамики поляризации конденсата, возбуждаемого на верхнем подуровне, за исключением его небольшой деполяризации, вызванной некогерентным рассеянием. В частности, уравнения описывают (i) поляризационную неустойчивость конденсата, сопровождающуюся сильными осцилляциями $\rho_{x \pm y}$ и $\rho_{c}$, (ii) соответствие периода осцилляций $\rho_{x \pm y}$ и $\rho_{c}$ в конденсате с низкой плотностью расщеплению поляритонного уровня в МР и уменьшение периода при малых $t$ из-за перенормировки резонансных часто поляритонов в плотном конденсате и, наконец, (iii) смену знака $\rho_{x, y}$ при больших $t$, являющуюся свидетельством перераспределения на нижний подуровень поляритонов, без какого-либо некогерентного рассеяния.

Для выяснения физической причины поляризационной неустойчивости конденсата и его постепенного когерентного перехода с верхнего подуровня на нижний рассмотрим более простую - нульмерную - спинорную систему со спин-анизотропным взаимодействием. Динамика двухкомпонентного поляритонного поля $\psi_{\sigma}(\mathbf{r})$ описывается нелинейным гамильтонианом

$$
i d \psi_{\sigma} / d t=H_{\sigma, \sigma^{\prime}} \psi_{\sigma^{\prime}}
$$

где

$H_{\sigma, \sigma^{\prime}} \psi_{\sigma^{\prime}}=H_{0, \sigma, \sigma^{\prime}} \psi_{\sigma^{\prime}}+\alpha_{1}\left|\psi_{\sigma}\right|^{2}+\left(\alpha_{2}\left|\psi_{-\sigma}\right|^{2}\right) \delta_{\sigma, \sigma^{\prime}}, H_{0, \sigma, \sigma^{\prime}}$ описывает поляризационное расщепление при нулевой плотности. Для описания матрицы $2 \times 2$ используем 

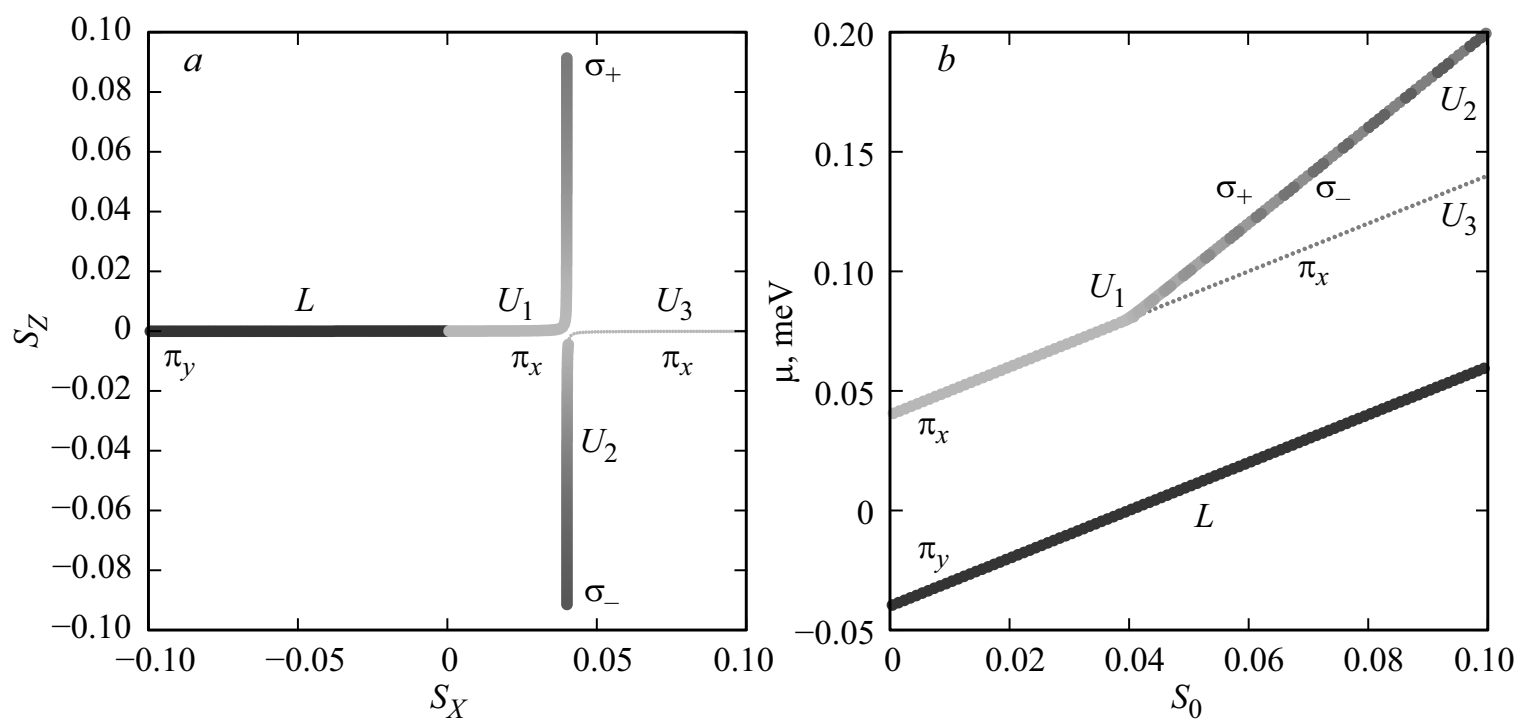

Рис. 7. (a) Рассчитанные зависимости стационарных спиновых состояний поляритонного конденсата от $S_{x}$ в MP с $\delta_{l}=0.08$ meV. Стабильные и неустойчивые состояния показаны толстыми и тонкими линиями, соответственно. $(b)$ Химические потенциалы стационарных состояний конденсата как функция его плотности, рассчитанные по тем же параметрам, что и на панели $(a)$. При $S_{0}>0.04$ ветвь $U_{1}$ идет выше $U_{2}$ на $\delta_{c}=0.1 \mu \mathrm{eV}$ и визуально совпадает с $U_{2}$.

вектор Стокса или псевдоспин, представляющий разложение матрицы по единичной матрице и матрицам Паули

$$
\rho_{\sigma, \sigma^{\prime}}=\left|\psi_{\sigma}\right\rangle\left\langle\psi_{\sigma^{\prime}}\right|=S_{0} \mathbf{1}+S_{x} \boldsymbol{\sigma}_{x}+S_{y} \boldsymbol{\sigma}_{y}+S_{z} \boldsymbol{\sigma}_{z},
$$

и гамильтониан, соответственно, в виде

$$
H_{\sigma, \sigma^{\prime}}=1 / 2\left(\Omega_{0} \mathbf{1}+\Omega_{x} \boldsymbol{\sigma}_{x}+\Omega_{y} \boldsymbol{\sigma}_{y}+\Omega_{z} \boldsymbol{\sigma}_{z}\right) .
$$

В частности, $S_{z}=\left(\left|\psi_{+}\right|^{2}-\left|\psi_{-}\right|^{2}\right) / 2 \quad$ и $\quad S_{0}=$ $=\left(\left|\psi_{+}\right|^{2}+\left|\psi_{-}\right|^{2}\right) / 2$ - длина псевдоспина. $\Omega_{y}$ можно занулить поворотом осей в $x y$-плоскости. В этом случае $\quad \boldsymbol{\Omega}=\left(\delta_{l}, 0, \delta_{c}+2\left(\alpha_{1}-\alpha_{2}\right) S_{z}\right) \quad$ и $\quad \Omega_{0}=H_{\sigma \sigma}^{0}$ $+2\left(\alpha_{1}+\alpha_{2}\right) S_{0}$, где $\delta_{c}$ и $\delta_{l}-$ зеемановское расщепление и расщепление линейно поляризованнных состояний в нулевом магнитном поле соответственно.

Используя уравнение фон Неймана для матрицы плотности $i \hbar \dot{\rho}=H \rho-\rho H$, получаем уравнение для псевдоспина

$$
\hbar d \mathbf{S} / d t=\mathbf{\Omega} \times S .
$$

В стационарном состоянии $\boldsymbol{\Omega} \times \mathbf{S}=0$, т. е. $\mathbf{S}$ параллелен или антипараллелен $\Omega$ и, следовательно,

$$
\delta_{l} / S_{x}=\delta_{c} / S_{z}+2\left(\alpha_{1}-\alpha_{2}\right) .
$$

Решениями этого уравнения являются 2 гиперболы в плоскости,$S_{x}-S_{z}$ “ с асимптотами $2 S_{x}^{\infty}=\delta_{l} /\left(\alpha_{1}-\alpha_{2}\right)$ и $2 S_{z}^{\infty}=-\delta_{c} /\left(\alpha_{1}-\alpha_{2}\right)$.

Все возможные стационарные спиновые состояния конденсата показаны на рис. 7, $a$. На рисунке видно, что есть четыре ветви, объединенные в две гиперболы на плоскости ,, $S_{x}-S_{z}$ “. Вблизи $S_{x}=0$ существуют две устойчивые ветви с противоположными псевдоспинами,
$L$ и $U_{1}$, соответствующие нижнему и верхнему поляритонным подуровням, показанным на рис. 7, $b$. Вблизи $S=0$ энергетическое расщепление между устойчивыми ветвями в нулевом магнитном поле равно $\delta_{l}$.

С ростом плотности на нижней ветви $\left(S_{x}<0\right)$ конденсат сохраняет линейную поляризацию $\pi_{y}$, тогда как увеличение плотности на верхней ветви $\left(S_{x}>0\right)$ приводит к превращению поляризации конденсата в циркулярную. Знак $\rho_{c}$ определяется знаком $\delta_{c}$, на рис. 7, $a$ показан случай $\delta_{c}>0$. Обе ветви этой гиперболы устойчивы. В дальнейшем в расчетах $\delta_{l}=80 \mu \mathrm{eV}, \delta_{c}=0.1 \mu \mathrm{eV}$, $\alpha_{1}=1 \mathrm{meV}$ и $\alpha_{2}=0$.

Вторая гипербола на рис. 7, $a$ делится на две ветви точкой с минимальной длиной псевдоспина $S_{\min }=\delta_{l} / 2\left(\alpha_{1}-\alpha_{2}\right)$. Эта величина определяет критическую плотность конденсата, выше которой возможны еще два стационарных состояния, $U_{2}$ и $U_{3}$. При этом только одна ветвь с поляризацией, приближающейся при высоких плотностях к круговой, $U_{2}$, является стабильной, другая $-U_{3}-$ с поляризацией, приближающейся к линейной $\pi_{x}$, оказывается неустойчивой и показана меньшими символами на рис. 7. Критическая длина псевдоспина стремится к бесконечности в системе с симметричным по спину межчастичным взаимодействием. Это означает, что наблюдаемая в эксперименте неустойчивость линейной поляризации в конденсате на верхнем поляритонном подуровне, обусловлена спиновой анизотропией поляритон-поляритонного взаимодействия.

Рассчитанная динамика псевдоспина при большой плотности конденсата с конечным временем жизни $\tau_{\mathrm{LP}}=15 \mathrm{ps}$, возбуждаемого на верхнем подуровне, показана на рис. 8. Толстые (тонкие) линии отображают эволюцию псевдоспина для $|\mathbf{S}|$ больше (меньше) $S_{\min }$. 


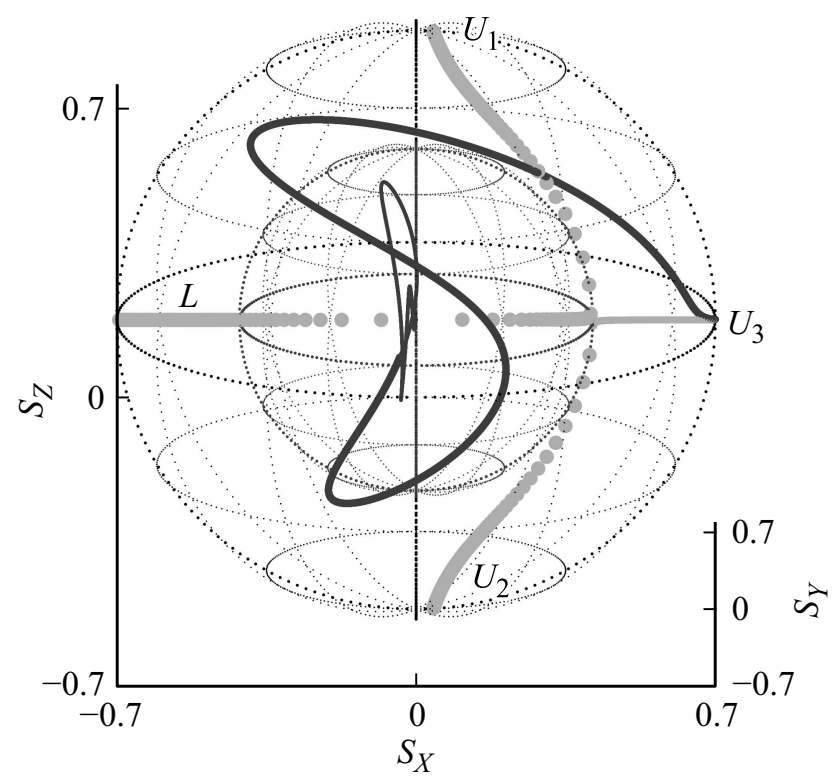

Рис. 8. Рассчитанная динамика прецессии псевдоспина с инверсией линейной поляризации для конечного времени релаксации. Жирные (тонкие) линии отображают эволюцию псевдоспина для $|\mathbf{S}|$ больше (меньше) $S_{\min }$. Длина псевдоспина логарифмически масштабирована (направление не изменяется). Радиус внешней черной сферы соответствует длине псевдоспина $|\mathbf{S}(t=0)|=0.7$, а внутренней сферы $-S_{\min }=0.04$. Стационарные состояния из рис. 7 показаны серыми линиями (они деформированы вследствие логарифмического представления).

Длина псевдоспина - логарифмическая шкала (направление неизмененное). Внешняя черная сфера соответствует $S_{0}=0.7$, а внутренняя соответствует $S_{\min }=0.04$. Стационарные состояния из рис. 7 показаны серыми линиями.

Рис. 8 показывает, что неустойчивость $\pi_{x}$-поляризованного состояния $U_{3}$ приводит к вращению псевдоспина $\mathbf{S}$ с одновременным уменьшением его амплитуды, сначала, вокруг стабильного состояния $U_{1}$ с частью траектории в области $S_{x}<0$, затем вокруг $U_{2}$ и снова попаданием в область отрицательного $S_{x}$, где амплитуда $\mathbf{S}$ становится уже меньше $S_{\min }$. Уменьшение длины $\mathbf{S}$ меньше $S_{\min }$ приводит к изменению его оси вращения с $U_{2}$ на $L$, что ведет к инверсии линейной поляризации. Система переходит в режим линейных джозефсоновских осцилляций с периодом, определяемым величиной расщепления поляритонных подуровней расслоением $\delta_{l}$. Таким образом, рассмотрение 0D модели показывает, что для инверсии поляризации, наблюдаемой в эксперименте при высоких $P$, некогерентное рассеяние не требуется.

Обсудим причину некогерентной инверсии поляризации. В конденсате с постоянной плотностью псевдоспин $\mathbf{S}$ после развития поляризационной неустойчивости движется по замкнутой траектории на сфере Блоха: сначала становится частично циркулярно поляризованным вследствие подмешивания состояний из нижнего состо- яния $\pi_{y}$ и затем возвращается в исходное состояние $\pi_{x}$. Наличие циркулярной компоненты приводит к увеличению полной энергии взаимодействия вследствие нелинейного вклада $\left(\alpha_{1}+\alpha_{2}\right) S_{0}^{2}+\left(\alpha_{1}-\alpha_{2}\right) S_{z}^{2}$, которое полностью компенсируется понижением линейного вклада, пропорционального $\delta_{l} S_{x}$, из-за подмешивания состояний с нижнего подуровня, в соответствии с законом сохранения энергии. Величина же химпотенциала $\mu$ при этом не остается постоянной из-за наличия квадратичного по $S_{z}$ вклада в энергию: $\mu$ минимален при линейной поляризации конденсата и растет с ростом $\rho_{c}$. В затухающем конденсате это приводит к тому, что исчезающие из конденсата частицы с эллиптической поляризацией в течение периода осцилляции $\rho_{c}$ уносят больше энергии, чем из линейно поляризованного конденсата за это же время. Возникающий дефицит компенсируется заменой в остающемся конденсате части $\pi_{x}$-компоненты компонентой $\pi_{y}$, имеющей меньшую энергию, без нарушения когерентности конденсата.

\section{5. Выводы}

Исследованы пространственная когерентность и поляризации свободно затухающего поляритонного конденсата, возбуждаемого резонансно на разных подуровнях нижней поляритонной ветви в МР с пониженной латеральной симметрией линейно поляризованными пикосекундными лазерными импульсами, не приводящими к возбуждению экситонного резервуара. Обнаружено, что конденсат, возбуждаемый как на нижнем, так и на верхнем подуровне, наследует когерентность лазерного импульса в широком диапазоне плотностей возбуждения и сохраняет ее в течение десятков пикосекунд. Поляризация же конденсата сохраняется только в конденсате на нижнем подуровне.

Линейно поляризованный конденсат, возбужденный на верхнем подуровне, теряет свою устойчивость при плотностях выше некоторого порогового значения: он переходит в режим внутренних джозефсоновских колебаний с сильно осциллирующими циркулярной и диагональной линейной степенями поляризации и постепенным увеличением в конденсате поляритонной компоненты с нижнего подуровня. При больших плотностях конденсата поляритон-поляритонное взаимодействие приводит к нелинейному эффекту Джозефсона.

Найдено, что все эффекты хорошо описываются в рамках спинорных уравнений Гросса-Питаевского. Показано, что причиной поляризационной неустойчивости конденсата является спиновая анизотропия поляритон-поляритонного взаимодействия $\left(\alpha_{l} \neq \alpha_{2}\right)$ : пороговая плотность для возникновения неустойчивости растет с уменьшением разности констант пропорционально отношению $\delta_{l} /\left(\alpha_{l}-\alpha_{2}\right)$. Увеличение в конденсате поляритонной компоненты с нижнего подуровня также связано со спиновой анизотропией поляритон-поляритонного взаимодействия. Неравенство $\alpha_{l}$ и $\alpha_{2}$ ведет к зависимо- 
сти химпотенциала конденсата от $\rho_{c}$ даже при постоянной плотности: $\mu$ минимален при линейной поляризации конденсата и растет с ростом $\rho_{c}$. В затухающем конденсате дефицит энергии, возникающий при исчезновение из конденсата частицы с эллиптической поляризацией, компенсируется заменой части компоненты с верхнего подуровня компонентой с нижнего подуровня с сохранением когерентности конденсата.

Авторы выражают благодарность С.С. Гаврилову, М.М. Глазову, С.Г. Тиходееву и В.Б. Тимофееву за плодотворные обсуждения.

\section{Список литературы}

[1] C. Weisbuch, M. Nishioka, A. Ishikawa, Y. Arakawa. Phys. Rev. Lett. 69, 3314 (1992).

[2] J. Kasprzak J., M. Richard, S. Kundermann, A. Baas, P. Jeambrun, J.M.J. Keeling, F.M. Marchetti, M.H. Szymánska, R. André, J.L. Staehli, V. Savona, P. Littlewood, B. Deveaud-Plédran, Le Si Dang. Nature (London) 443, 409 (2006).

[3] R. Balili, V. Hartwell, D. Snoke, L. Pfeiffer, K. West. Science 316, 1007 (2007).

[4] A.V. Kavokin, J.J. Baumberg, G. Malpuech, F.P. Laussy. Microcavities. Oxford University Press, Oxford (2007).

[5] D. Sanvitto, V. Timofeev. Exciton Polaritons in Microcavities. Springer-Verlag, Berlin (2012).

[6] A. Bramati, M. Modugno. Physics of Quantum Fluids. Springer-Verlag, Berlin (2013).

[7] S.S. Gavrilov, A.V. Sekretenko, S.I. Novikov, C. Schneider, S. Höfling, M. Kamp, A. Forchel, V.D. Kulakovskii. Appl. Phys. Lett. 102, 011104 (2013).

[8] H. Deng, G.S. Solomon, R. Hey, K.H. Ploog, Y. Yamamoto. Phys. Rev. Lett. 99, 126403 (2007).

[9] G. Nardin, K.G. Lagoudakis, M. Wouters, M. Richard, A. Baas, R. André, Le Si Dang, B. Pietka, B. Deveaud-Plédran. Phys. Rev. Lett. 103, 256402 (2009).

[10] H. Ohadi, E. Kammann, T.C. H.Liew, K.G. Lagoudakis, A.V. Kavokin, P.G. Lagoudakis. Phys. Rev. Lett. 109, 016404 (2012).

[11] M. Steger, G. Liu, B. Nelsen, C. Gautham, D.W. Snoke, R. Balili, L. Pfeiffer, K. West. Phys. Rev. B 88, 235314 (2013).

[12] A. Amo A., J. Lefrére, S. Pigeon, C. Adrados, C. Ciuti, L. Carusotto, R. Houdré, E. Giacobino, A. Bramati. Nature Phys. 5, 805 (2009).

[13] A.V. Larionov, V.D. Kulakovskii, S. Höfling, C. Schneider, L. Worschech, A. Forchel. Phys. Rev. Lett. 105, 256401 (2010).

[14] K.G. Lagoudakis, M. Wouters, M. Richard, A. Baas, I. Carusotto, R. André, Le Si Dang, B. Deveaud-Plédran. Nature Phys. 4, 706 (2008).

[15] K.G. Lagoudakis, B. Pietka, M. Wouters, R. André, B. Deveaud-Plédran. Phys. Rev. Lett. 105, 120403 (2010).

[16] V.V. Belykh, N.N. Sibeldin, V.D. Kulakovskii, M.M. Glazov, M.A. Semina, C. Schneider, S. Höfling, M. Kamp, A. Forchel. Phys. Rev. Lett. 110, 137402 (2013).

[17] D.A. Mylnikov, V.V. Belykh, N.N. Sibeldin, V.D. Kulakovskii, C. Schneider, S. Höfling, M. Kamp, A. Forchel. JETP. Lett. 101, 513 (2015).
[18] J. Schmutzler, T. Kazimierczuk, O. Bayraktar, M. Assmann, M. Bayer, S. Brodbeck, M. Kamp, C. Schneider, S. Höfling. Phys. Rev. B 89, 115119 (2014).

[19] N.A. Gippius, I.A., Shelykh, D.D. Solnyshkov, S.S. Gavrilov, Y. Rubo, A.V. Kavokin, S.G. Tikhodeev, G. Malpuech. Phys. Rev. Lett. 98, 236401 (2007).

[20] I.A. Shelykh, T.C.H. Liew, A.V. Kavokin. Phys. Rev. Lett. 100, 116401 (2008).

[21] T.K. Paraïso, M. Wouters, Y. Leger, F. Morier-Genoud, B. Deveaud-Plédran. Nature Mater. 9, 655 (2010).

[22] S.S. Gavrilov, A.V. Sekretenko, N.A. Gippius, C. Schneider, S. Höfling, M. Kamp, A. Forchel, V.D. Kulakovskii. Phys. Rev. B 87, 201303(R) (2013).

[23] A.V. Sekretenko, S.S. Gavrilov, V.D. Kulakovskii. Phys. Rev. B 88, 195302 (2013).

[24] M. Vladimirova, S. Cronenberger, D. Scalbert, K.V. Kavokin, A. Miard, A. Lemaítre, J. Bloch, D. Solnyshkov, G. Malpuech, A.V. Kavokin. Phys. Rev. B 82, 075301 (2010).

[25] D.N. Krizhanovskii, S.S. Gavrilov, A.P.D. Love, D. Sanvitto, N.A. Gippius, S.G. Tikhodeev, V.D. Kulakovskii, D.M. Whittaker, M.S. Skolnick, J.S. Roberts. Phys. Rev. B 77, 115336 (2008).

[26] S.S. Gavrilov, A.S. Brichkin, A.A. Demenev, A.A. Dorodnyy, S.I. Novikov, V.D. Kulakovskii, S.G. Tikhodeev, N.A. Gippius. Phys. Rev. B 85, 075319 (2012).

[27] A.V. Yulin, O.A. Egorov, F. Lederer, D.V. Skryabin. Phys. Rev. A 78, 061801(R) (2008).

[28] M. Sich, D.N. Krizhanovskii, M.S. Skolnick, A.V. Gorbach, R. Hartley, D.V. Skryabin, E.A. Cerda-Mendez, K. Biermann, R. Hey, P.V. Santos. Nature Photon. 6, 50 (2012).

[29] G. Slavcheva, A.V. Gorbach, A. Pimenov, A.G. Vladimirov, D.V. Skryabin. Opt. Lett. 40, 1787 (2015)

[30] C. Adrados, A. Amo, T.C.H. Liew, R. Hivet, R. Houdre, E. Giacobino, A.V. Kavokin, A. Bramati. Phys. Rev. Lett. 105, 216403 (2010).

[31] A.A. Demenev, Ya.V. Grishina, S.I. Novikov, V.D. Kulakovskii, C. Schneider, S. Höfling. Phys. Rev. B 94, 195302 (2016).

[32] D.N. Krizhanovskii, E.A. Cerda-Méndez, S.S. Gavrilov, D. Sarkar, K. Guda, R. Bradley, P.V. Santos, R. Hey, K. Biermann, M. Sich, F. Fras, M.S. Skolnick. Phys. Rev. B 87, 155423 (2013).

Редактор Т.Н. Василевская 